Conservative mixing, competitive mixing and their applications

This article has been downloaded from IOPscience. Please scroll down to see the full text article.

2010 Phys. Scr. 2010014054

(http://iopscience.iop.org/1402-4896/2010/T142/014054)

View the table of contents for this issue, or go to the journal homepage for more

Download details:

IP Address: 130.102.42.98

The article was downloaded on 03/01/2011 at 05:30

Please note that terms and conditions apply. 


\title{
Conservative mixing, competitive mixing and their applications
}

\author{
A Y Klimenko \\ The University of Queensland, School of Mechanical and Mining Engineering, Brisbane, QLD 4072, \\ Australia \\ E-mail: klimenko@mech.uq.edu.au
}

Received 29 January 2010

Accepted for publication 24 March 2010

Published 31 December 2010

Online at stacks.iop.org/PhysScr/T142/014054

\begin{abstract}
In many of the models applied to simulations of turbulent transport and turbulent combustion, the mixing between particles is used to reflect the influence of the continuous diffusion terms in the transport equations. Stochastic particles with properties and mixing can be used not only for simulating turbulent combustion, but also for modeling a large spectrum of physical phenomena. Traditional mixing, which is commonly used in the modeling of turbulent reacting flows, is conservative: the total amount of scalar is (or should be) preserved during a mixing event. It is worthwhile, however, to consider a more general mixing that does not possess these conservative properties; hence, our consideration lies beyond traditional mixing. In non-conservative mixing, the particle post-mixing average becomes biased towards one of the particles participating in mixing. The extreme form of non-conservative mixing can be called competitive mixing or competition: after a mixing event, the loser particle simply receives the properties of the winner particle. Particles with non-conservative mixing can be used to emulate various phenomena involving competition. In particular, we investigate cyclic behavior that can be attributed to complex competing systems. We show that the localness and intransitivity of competitive mixing are linked to the cyclic behavior.
\end{abstract}

PACS numbers: 47.27.- $\mathrm{i}, 47.10 . \mathrm{A}-$

\section{Introduction}

Notional or stochastic particles are widely used as effective modeling tools in Lagrangian simulations of turbulent reacting flows as repeatedly reviewed in major publications [1-8]. These particles possess a set of properties altered by reactions and mixing between particles. The particles move with a fluid flow and, as introduced in [9], are also engaged in a random walk. The emergence of sparse-Lagrangian methods $[10,11]$ released the particles from being constrained by Eulerian grid cells. Recent developments seem to indicate that the stochastic particles, which were introduced into wide use by Pope's seminal work $[1]^{1}$, represent fundamental blocks that can be used to construct various types of models. Stochastic particles possess properties that can be exchanged due to interaction between particles (i.e. mixing) and their motions involve both deterministic and stochastic components. The models using particles correspond to complex systems that can be divided

1 In [12], these particles are suggested to be called Pope particles. into a large number of semi-autonomous but interacting units emulated by particles. Stochastic particles represent prime objects that combine three fundamental processes of convection, random walk and interaction between particle properties.

While the particles seem to represent a general modeling tool, conventional mixing is subject to obvious restrictions - this mixing is conservative and non-competitive. This means that the overall amount of each scalar is preserved and contribution of each particle to the state of the particles after mixing is guaranteed. The use of this type of mixing in turbulent combustion modeling is determined by the properties of turbulent scalar transport [1] and is justified. At the same time, omitting these restrictions can result in more general models corresponding to a wider class of natural phenomena. In the present work, we look beyond conventional mixing but consider only two-particle mixing, i.e. interactions between many particles that can be represented as a superposition of interactions between selected couples. Investigation of conventional conservative 
mixing in [13] indicates that two-particle interactions are sufficiently general for adequate modeling.

The mixing considered in the present work is competitive. This means that particle contributions to the states after mixing are not guaranteed-there are winners and losers among the particles. The competitive mixing schemes allow us to investigate the properties of competition in its most abstract form and determine most generic features of competing systems. We stress that analysis or modeling of realistic competing systems (biological, economical, etc) is not within the scope of this work and the presented simulations are not intended to simulate any specific event or process. These simulations, however, examine (and provide qualifications for) the hypothesis of commonality of cyclic behavior in complex competing systems [14].

\section{Competitive mixing}

In this work, we consider two-particle mixing-only two particles are allowed to mix at a given moment. During the mixing process, say of particles 1 and 2, the particle properties $Y^{(1)}$ and $Y^{(2)}$ are changed according to

$$
\left[\begin{array}{l}
\hat{Y}^{(1)} \\
\hat{Y}^{(2)}
\end{array}\right]=\left[\begin{array}{ll}
M^{(11)} & M^{(12)} \\
M^{(21)} & M^{(22)}
\end{array}\right]\left[\begin{array}{l}
Y^{(1)} \\
Y^{(2)}
\end{array}\right],
$$

where the properties of mixing are determined by the matrix $\mathbb{M}$. Each particle $p$ may, of course, possess several properties $Y_{1}^{(p)}, Y_{2}^{(p)}, \ldots$; if this is the case, mixing operation (1) is applied to each of the properties. Different choices of the matrix $\mathbb{M}$ result in mixing with different properties. Conventional mixing can be described with the use of the matrices

$$
\mathbb{M}_{1}=\left[\begin{array}{ll}
1 & 0 \\
0 & 1
\end{array}\right], \quad \mathbb{M}_{2}=\left[\begin{array}{ll}
0 & 1 \\
1 & 0
\end{array}\right],
$$

representing identical mixing $\mathbb{M}_{1}$, which does not change the particle properties, and swap-mixing matrix $\mathbb{M}_{2}$, which swaps the properties between the particles. With the use of these matrices, conventional mixing may be represented by

$$
\mathbb{M}=(1-\alpha) \mathbb{M}_{1}+\alpha \mathbb{M}_{2},
$$

where $\alpha$ is the extent of mixing: $\alpha=0$ corresponds to no mixing, $\alpha=1 / 2$ corresponds to complete mixing and $\alpha=1$ corresponds to swapping properties. Mixing specified by (2) is referred to as Curl's mixing [15] and its properties are comprehensively discussed in publications dedicated to Lagrangian modeling of turbulent reacting flows [1, 3, 7, 8, 13]. Conventional mixing (2) is

1. Non-discriminative $M^{(p q)}=M^{(q p)} ;$ mixing treats particles 1 and 2 in the same way.

2. Conservative $\sum_{p} M^{(p q)}=1$; mixing preserves the total values $\dot{Y}^{(1)}+\dot{Y}^{(2)}=Y^{(1)}+Y^{(2)}$.

3. Non-segregating $\sum_{q} M^{(p q)}=1$; mixing preserves the so-called well-mixed conditions; that is, if $Y^{(1)}=Y^{(2)}$, then $Y^{(1)}=\dot{Y}^{(2)}$.

4. Bounded; mixing preserves boundaries of the values $Y^{(p)}$ provided $0 \leqslant \alpha \leqslant 1$.
5. Dissipative; mixing reduces differences between $Y^{(p)}$ provided $0<\alpha<1$.

Note that not all of these properties are independent-for example, the non-discriminative and non-segregating character of mixing ensures its conservativeness.

Conventional mixing is successfully used in modeling dissipative mixing such as scalar transport and dissipation in turbulent flows. The non-discriminating character of conventional mixing ensures that in each mixing couple, particle 1 affects particle 2 in the same way as particle 2 affects particle 1 . In the present work, we consider competitive mixing that may discriminate between particles but only in a certain 'fair' way. In competitive mixing, particles are not equal-there is a winner and there is a loser. Competition is the process of determining the winner and the loser, while competitive mixing is redistribution of the particle properties on the basis of the competition outcomes.

We consider competitive mixing represented by the following mixing matrices:

$$
\mathbb{M}_{3}=\left[\begin{array}{ll}
1 & 0 \\
1 & 0
\end{array}\right], \quad \mathbb{M}_{4}=\left[\begin{array}{ll}
0 & 1 \\
0 & 1
\end{array}\right] .
$$

If $\mathbb{M}_{3}$ is used particle 1 is the winner while particle 2 is the loser, and vice versa for matrix $\mathbb{M}_{4}$. The winner propagates information represented by its properties to the loser, while properties of the loser are lost. One can interpret this as the winner capturing the resource of the loser and using it to replicate itself. This mixing is not conservative (information is duplicated), but it is non-segregating and dissipative as differences between particles are reduced as the result of mixing. For the matrix $\mathbb{M}=(1-\alpha) \mathbb{M}_{3}+\alpha \mathbb{M}_{4}$ the values $\alpha=0$ and $\alpha=1$ correspond to particles 1 and 2 winning the competition while there is conventional mixing and no competition for $\alpha=1 / 2$. A softer version of competition can be introduced by $\alpha$ deviating from $1 / 2$ but not reaching 0 or 1. In the present work we, however, restrict our consideration to pure competition $(\alpha=0,1)$ when the winner completely dominates the loser. With introduction of the competition index $I=2 \alpha-1$ so that

$$
\mathbb{M}=\frac{1+I}{2} \mathbb{M}_{3}+\frac{1-I}{2} \mathbb{M}_{4}
$$

the outcome of the competitive mixing is given by

$$
\begin{array}{ll}
\dot{Y}^{(1)}=Y^{(1)}, & \dot{Y}^{(2)}=Y^{(1)}, \quad \text { when } \quad Y^{(1)} \succ Y^{(2)}, \quad I=1, \\
\dot{Y}^{(1)}=Y^{(2)}, & \dot{Y}^{(2)}=Y^{(2)}, \quad \text { when } \quad Y^{(1)} \prec Y^{(2)}, \quad I=-1 .
\end{array}
$$

The symbol ' $\mathrm{A} \succ \mathrm{B}$ ' denotes that $\mathrm{A}$ is the winner in competition with $\mathrm{B}$ and this notation is generally different from the conventional arithmetic comparison $A>B$. The competition considered in the present work is 'merit-based'-the winner and the loser are determined only on the basis of the particle properties before mixing. In principle, it is possible to consider more complicated competitions where the outcome is determined not only by the properties of the particles but also by other factors (physical location, for example). Sometimes we may have to deal with situations when no winner can be determined $(\mathrm{A} \sim \mathrm{B})$. In this case particles may be left with their initial 
properties or the winner can be determined randomly. In many cases, when the probability of $\mathrm{A} \sim \mathrm{B}$ is zero, the exact treatment of competitive equivalence does not matter. Another possible generalization, which may resemble modification of Curl's mixing model $[16,17]$, is random selection of the winner for all mixing couples with probability determined by competition. In reacting flows, non-conservative mixing can be interpreted as the joint influence of physical mixing (which is conservative) and a premixed source term (which generates or destroys scalars). The simplest example of competitive mixing is given by particles of two kinds: the losers with $Y=0$ and the winners with $Y=1$. Mixing of a particle with $Y=0$ with a particle with $Y=1$ results in two particles with $Y=1$, while any other mixing combination does not alter particle values.

In the conservative version of competitive mixing, the matrix $\mathbb{M}$ is given by

$$
\mathbb{M}_{5}=\left[\begin{array}{ll}
1 & 1 \\
0 & 0
\end{array}\right], \quad \mathbb{M}_{6}=\left[\begin{array}{ll}
0 & 0 \\
1 & 1
\end{array}\right] .
$$

Mixing (6) specifies redistribution of a limited resource between the particles in a mixing couple favoring the winner and discriminating the loser-the winner acquires properties of the loser, while the loser loses everything. This mixing is segregating and creates inequalities. In the present work, however, our consideration is restricted to non-conservative and non-segregating mixing specified by (5) and this mixing is implied when referred to as competitive mixing.

\section{Transitive competition}

Each particle $p$ possesses a set of properties particle properties $\mathbf{y}^{(p)}=y_{1}^{(p)}, y_{2}^{(p)}, \ldots, y_{n_{d}}^{(p)}$ from a given $n_{d}$-dimensional domain $G$ and the outcomes of the competition are decided on the basis of these properties. Competition between particles presumes that for any two particles $p$ and $q$, one of the following relations is valid: $\mathbf{y}^{(p)} \precsim \mathbf{y}^{(p)}$ or $\mathbf{y}^{(p)} \succsim \mathbf{y}^{(p)}$. This means that the particles are totally pre-ordered either by selecting the winner and the loser or, in some cases, by determining that the particles are equivalent: $\mathbf{y}^{(p)} \prec \mathbf{y}^{(q)}$ or $\mathbf{y}^{(p)} \succ \mathbf{y}^{(q)}$ or $\mathbf{y}^{(p)} \simeq \mathbf{y}^{(q)}$. The competition considered in this section is transitive, which means that

$$
\mathbf{y}^{(p)} \precsim \mathbf{y}^{(q)} \quad \text { and } \quad \mathbf{y}^{(q)} \precsim \mathbf{y}^{(r)} \Rightarrow \mathbf{y}^{(p)} \precsim \mathbf{y}^{(r)}
$$

for any three particles $p, q$ and $r$. As discussed in the appendix, transitive competition can be equivalently or nearly equivalently represented by a ranking function $R(\mathbf{y})$ :

$$
\mathbf{y}^{(p)} \precsim \mathbf{y}^{(q)} \Leftrightarrow R\left(\mathbf{y}^{(p)}\right) \leqslant R\left(\mathbf{y}^{(q)}\right) .
$$

Using the competition indicator $I^{(p q)}$, this relation can be written as

$$
I^{(p q)}=\operatorname{sign}\left(R\left(\mathbf{y}^{(p)}\right)-R\left(\mathbf{y}^{(q)}\right)\right)
$$

so that

$$
\begin{array}{ll}
\mathbf{y}^{(p)} \prec \mathbf{y}^{(q)} & \text { if } I^{(p q)}<0 \\
\mathbf{y}^{(p)} \simeq \mathbf{y}^{(q)} & \text { if } I^{(p q)}=0 \\
\mathbf{y}^{(p)} \succ \mathbf{y}^{(q)} & \text { if } I^{(p q)}>0
\end{array}
$$

where $\operatorname{sign}(\zeta)$ denotes the signum function, which is +1 for $\zeta>0,-1$ for $\zeta<0$ and 0 for $\zeta=0$. Note that the sign function can be omitted or replaced by another similar function. By definition, the competition indicator is an antisymmetric function $I^{(p q)}=-I^{(q p)}$. Assuming that the measure $\mu$ defining probability $\mathbb{P}$ is specified on $G$, a suitable ranking can simply be introduced by

$$
R_{\mu}\left(\mathbf{y}^{\circ}\right)=\mathbb{P}\left(\mathbf{y}^{(\mu)} \precsim \mathbf{y}^{\circ}\right),
$$

where $\mathbf{y}^{(\mu)}$ is distributed according to the measure $\mu$, which is called the reference measure. In most cases we link this measure to the probability distribution of mutations considered in following sections. The superscript ' $\mu$ ' can be omitted when the uniform reference measure is implied. Practically, the existence of equivalent or nearly equivalent ranking for transitive competition means that we do not need to trace the complete set of particle properties $\mathbf{y}$. Instead, we assign $Y=R_{\mu}(\mathbf{y})$ to each particle and the competition outcomes are fully determined by the particle values $Y^{(p)}$. The value $Y$ is bounded $0 \leqslant Y \leqslant 1$ since $\mu(G)=1$ for any probability measure. If $Y(\mathbf{y})$ is randomly generated by distributing $\mathbf{y}$ on $G$ with the probability density determined by the measure $\mu$, then $Y$ is uniformly distributed on the interval $0 \leqslant Y \leqslant 1$.

Competition between particles possessing a single property $0 \leqslant Y^{(p)} \leqslant 1$ is now considered. There is no localization in physical space and at every time step, $n_{p}$ particles form $n_{p} / 2$ mixing couples at random. The particle distribution is characterized by the cumulative probability $F(Y)$ and the pdf $f(Y)$

$$
F\left(Y^{\circ}\right)=\mathbb{P}\left(Y^{(*)} \leqslant Y^{\circ}\right), \quad f\left(Y^{\circ}\right)=\frac{\mathrm{d} F}{\mathrm{~d} Y^{\circ}} .
$$

The superscript ' $(*)$ ' indicates the current distribution of the particles that may be different from the reference measure. In general, the function $F(Y)$ can be discontinuous; in this case, the left-hand side limit $F(Y)^{-}$may not be the same as the right-hand side limit $F(Y)^{+}$of the same function and the pdf $f(Y)$ has generalized components.

\subsection{Competition without mutations}

The probability that particle $p$ is a winner in competition with any other particle selected at random is given by the fraction of particles with $Y<Y^{(p)}$, that is, $F\left(Y^{(p)}\right)^{-}$plus $\left(F\left(Y^{(p)}\right)^{+}-F\left(Y^{(p)}\right)^{-}\right) / 2$ - half of the fraction of particles of equivalent $\operatorname{rank} Y \simeq Y^{(p)}$, assuming that the winner is chosen at random in competition of equivalent particles. If $F(Y)$ is continuous, then the distributions after mixing become

$$
f^{\prime}(Y)=2 f(Y) F(Y) \Longrightarrow \dot{F}(Y)=F^{2}(Y) .
$$

Here the winners distributed according to the pdf of $f(Y) F(Y)$ retain their distribution, while the losers distributed according to $f(Y)(1-F(Y))$ change their distribution to that of the winners. The function $F(Y)$ is determined by integrating $f(Y)$. At each time step the cumulative function experiences the following change:

$$
\Delta F \equiv \dot{F}(Y)-F(Y)=-F(1-F) .
$$


Assuming that the initial distribution of the particles is continuous, the cumulative function evolves towards its steady state. If $\Delta F=0$, then only two steady values are possible for $F: 0$ and 1 . The values of the cumulative function at the consecutive time steps are given by equation (13): $F, F^{2}, F^{4}$, $F^{8}, \ldots$. At this point we stress the importance of the leading particle, which, among other particles, has the maximal value of $Y_{*}$. The subscript ' $*$ ' is used here and further in the paper to denote values linked to the leading point. It is clear that no other particle can gain a value above $Y_{*}$ and that $Y_{*}$ cannot be reduced since at least one particle that has $Y^{(p)}=Y_{*}$ always remains as a winner. The maximal value

$$
Y_{*}=\max _{p}\left(Y^{(p)}\right)
$$

remains constant during the form of competitive mixing considered here. The final distribution for this competition is given by

$$
F(Y)=\left\{\begin{array}{ll}
1, & Y \geqslant Y_{*} \\
0, & Y<Y_{*}
\end{array}\right\}
$$

\subsection{Competition with mutations}

The competition process becomes more sophisticated when mutations are introduced into consideration. The properties of the winner remain without change but these properties can experience some variations while being copied from the winner to the loser. These variations can be small but their presence is most important for the evolution of the system. Assuming that $Y^{(p)} \prec Y^{(q)}$ we put after the mixing

$$
\dot{Y}^{(p)}=(1-m) Y^{(q)}+m \zeta, \quad Y^{(p)} \prec Y^{(q)} ;
$$

here $m$ is the mutation level and $\zeta$ is a random value uniformly distributed in the interval $0 \leqslant \zeta \leqslant 1$ and generated independently for each mixing couple. Note that these mutations preserve the boundness $0 \leqslant Y \leqslant 1$. If $m=0$, there are no mutations. If $m=1$, the properties of the losers are randomly regenerated. This mutation can be considered as mixing of the property $Y$ with the randomly generated property $\zeta$ according to (2) with $\alpha=m$, and an $m$ th fraction of $Y$ is replaced by $\zeta$. Practically, the cases when mutations are present but their level $m$ is relatively small are of most interest.

One can consider a random walk that is generated by

$$
Y_{t+\Delta t}=(1-m) Y_{t}+m \zeta=Y_{t}-m\left(Y_{t}-\frac{1}{2}\right)+m \zeta^{\prime}
$$

where $\zeta^{\prime}=\zeta-\langle\zeta\rangle=\zeta-1 / 2$ and $\left\langle\left(\zeta^{\prime}\right)^{2}\right\rangle=1 / 12$. The long-time asymptotics of this random walk is represented by a diffusional process with drift $u=-m(Y-1 / 2) / \Delta t$ and diffusion coefficient $D=m^{2} /(24 \Delta t)$. The steady-state asymptote of the diffusion process is Gaussian, with moments given by $\left\langle Y_{t}\right\rangle=1 / 2, \quad\left\langle y_{t}^{2}\right\rangle=m / 24, \quad y_{t}=Y_{t}-\left\langle Y_{t}\right\rangle$. The dispersion of the steady-state distribution is small when $m \rightarrow 0$. Note that the diffusional approximation of this process is not bounded while the boundness of the random walk $(18)$ is preserved: $0 \leqslant Y_{t} \leqslant 1$. This seems to be of little importance as the pdf of the process is very small at the boundaries when $m$ is small. The case of competitive mixing is, however, highly dependent on the position of the leading particle and this particle may approach the boundary $Y=1$ where the diffusion approximation is incorrect and an alternative treatment of the problem is needed. We note significant differences between conventional and competitive mixing. If the number of particles is large, conventional mixing is not strongly affected by a single particle. However, a small group of particles or even a single particle, which is called 'leading' here, can exercise a disproportionately strong influence on the rest of the particles and alter or constrain the behavior of the whole system of particles in the case of competitive mixing. It seems that competitive mixing shares similarities more with premixed combustion than with non-premixed combustion-leading points can play an important role in turbulent premixed combustion [2].

Equation (17) can be rewritten in the form

$$
\dot{Y}^{(p)}=-m\left(1-\zeta-\left(1-Y^{(q)}\right)\right)+Y^{(q)}, \quad Y^{(p)} \prec Y^{(q)} .
$$

If $Y_{*}$ is the leading (i.e. maximal) value at a fixed moment, we can define $Z^{(q)}$ by $Y^{(q)}=Y_{*}+m Z^{(q)}$ (i.e. $Z^{(q)} \leqslant 0$ ), take into account that $1-\zeta$ is stochastically equivalent to $\zeta$ and rewrite

$$
\dot{Z}^{(p)} \approx-\zeta+y_{*}+Z^{(q)}, \quad Z^{(p)} \prec Z^{(q)},
$$

where $y_{*}=1-Y_{*}$ is introduced and the term $m Z$ is neglected, assuming that $m$ is small. The variable $Z$ is introduced to analyze the vicinity of the leading point.

The solution for competitive transitive mixing with mutations is obtained below by considering problems of progressively increasing complexity. This solution cannot and does not use the diffusion approximation for the random walk induced by mutations.

We consider first the case when $y_{*}=0$ in (20). This means that either $Y_{*}=1$ or $y_{*}$ is small and can be neglected. The effect of mutations on particle $p$ that has lost competition to particle $q$ is given by

$$
\dot{Z}^{(p)}=Z^{(q)}-\zeta, \quad Z^{(p)} \prec Z^{(q)} .
$$

Note that with this type of mutation no particle can overtake the leading particle and $Y_{*}$ remains constant. The equation for the steady distribution $F(Z)=\mathscr{F}(Z)$ is given by

$$
\frac{\widehat{\mathbb{T}}_{0} F^{2}(Z)}{2}+\frac{F^{2}(Z)}{2}=F(Z),
$$

where operator $\widehat{\mathbb{T}}_{0}$ introduces a random shift directed towards $Z=-\infty$ :

$$
\widehat{\mathbb{T}}_{0}(\varphi(Z))=\int_{Z}^{Z+1} \varphi\left(Z^{\circ}\right) \mathrm{d} Z^{\circ} .
$$

By differentiating this equation, one can easily obtain

$$
\frac{\mathrm{d} F(Z)}{\mathrm{d} Z}(1-F(Z))=\frac{F^{2}(Z+1)-F^{2}(Z)}{2} ;
$$

considering that $Z=0$ is the leading point with no particles beyond it, $F=1$ for $Z \geqslant 0$. The equation can be integrated for $-1 \leqslant Z \leqslant 0$ resulting in $F=2 \exp (Z / 2)-1$. The solution for $Z<-1$ is determined numerically by solving differential equation (24). The moments for this distribution are $\bar{Z} \equiv$ $\left\langle Z^{(p)}\right\rangle \approx-0.64,\left\langle\left(Z^{(p)}\right)^{2}\right\rangle \approx 0.6$. The evaluated curve is shown by circles in figure 1 , while the solid line represents a stochastic simulation involving 100000 particles. 


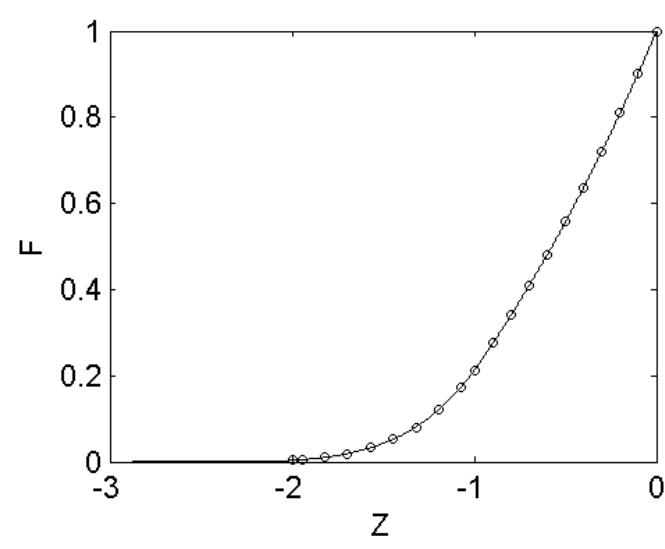

Figure 1. Cumulative distribution $F(Z)$ : ०, analytical/numerical solution; - , simulation using 100000 particles.

The obtained solution demonstrates that in spite of the mutational drift towards $Y=1 / 2$, the competing system of particles is located close to the leading point:

$$
\left\langle Y^{(p)}\right\rangle=Y_{*}+m \bar{Z}=Y_{*}+2 \bar{Z}\langle m \zeta\rangle .
$$

Note that $\bar{Z}<0$. Smaller mutation rates result in smaller deviations from the leading point.

\subsection{Competitive escalation}

The question whether the system of particles can move towards $Y=1$ still needs to be investigated. The fact that $y_{*}>0$ when $Y_{*}<1$ needs to be taken into account. Equation (22a) should be modified for this case and takes the form

$$
\frac{\widehat{\mathbb{T}} F^{2}(Z)}{2}+\frac{F^{2}(Z)}{2}=F(Z-U),
$$

where

$$
\widehat{\mathbb{T}}(\varphi(Z))=\int_{Z-y_{*}}^{Z+1-y_{*}} \varphi\left(Z^{\circ}\right) \mathrm{d} Z^{\circ}=\widehat{\mathbb{T}}_{0}\left(\varphi\left(Z-y_{*}\right)\right)
$$

and $U$ is the normalized rate of competitive escalation (i.e. increase in ranking per time step). This velocity is related to the physical velocity in $Y$-space by $u=U m / \Delta t$.

We can treat $y_{*}$ as a small parameter. The solution obtained in the previous subsection is denoted by $F_{0}(Z)$. We note that substitution of $F=F_{0}+y_{*} F_{1}+\cdots$ into (25) with subsequent linearization (retaining only terms linear in $y_{*}$ and $U$ ) does not yield a solution due to singularity at the leading point. This indicates the need for analysis of the vicinity of the leading point and, as we will see below, that $U$, which can be represented by the expansion $U=y_{*} U_{1}+$ $y_{*}^{2} U_{2}+\cdots$, has zero leading order term $U_{1}=0$ and $U \sim$ $y_{*}^{2}$ that cannot be determined from the first-order analysis. Finding $U$ requires consideration of the leading group of particles at a higher order.

We consider the vicinity of the leading point where $\phi=$ $1-F$ is small and equation (25) can be written as

$$
\widehat{\mathbb{T}} \phi(Z)+\phi(Z)=\phi(Z-U),
$$

where the terms $\phi^{2}$ are neglected. Assuming that the solution can be represented by an exponent $\phi(Z)=a \exp (-Z / b)$

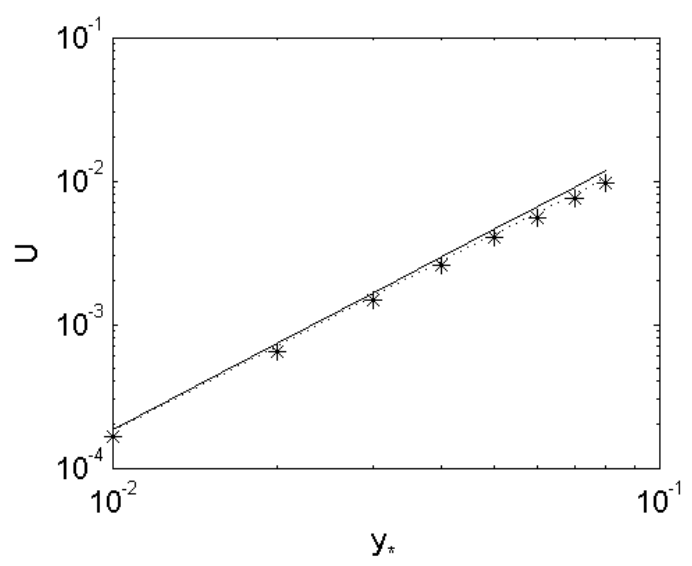

Figure 2. Rate of competitive escalation for transitive competition: *, simulation using 100000 particles; - , equation (31); - - -, equation (31) without exponent expansion.

where $\alpha$ and $b$ are small (of the order of $y_{*}$ ), we obtain the equation, which after dividing by $\phi(Z)$ becomes

$$
b \exp \left(\frac{y_{*}}{b}\right)-b \exp \left(\frac{y_{*}-1}{b}\right)+1=\exp \left(\frac{U}{b}\right) .
$$

This equation can be transformed into

$$
b \exp \left(\frac{y_{*}}{b}\right)=\exp \left(\frac{U}{b}\right)-1,
$$

where the term $\exp (-1 / b)$ is exponentially small and is neglected. Since $U \sim y_{*}^{2}$, the right-hand side exponent can be expanded into a series $\exp (U / b)=1+U / b+\cdots$, resulting in

$$
\frac{y_{*}}{b}+2 \ln (b)-\log (U)=0 .
$$

If $U$ is excessively small, no $b$ can be found to satisfy this equation. We are looking for the smallest possible value of $U$ that allows for a stationary solution. Differentiating this equation results in $y_{*} / b^{2}=2 / b$; hence $b=y_{*} / 2$. The corresponding value of $U$ is given by

$$
U_{*}=\exp (2) \frac{y_{*}^{2}}{4}, \quad u_{*} \equiv\left\langle\frac{\mathrm{d} Y_{*}}{\mathrm{~d} t}\right\rangle=\frac{m}{\Delta t} U_{*} .
$$

Figure 2 demonstrates that the rate of competitive escalation given by (31) is in good agreement with the results of stochastic simulations.

\subsection{Estimations for localized competition}

Assume that mutation for a mixing couple with loser $p$ and winner $q$ is given by

$$
\dot{Y}^{(p)}=Y^{(q)}-\xi, \quad Y^{(p)} \prec Y^{(q)},
$$

where $\xi$ is a random value. Assuming that $\xi$ is a positive or mostly positive value, we can write by analogy with the previous consideration

$$
\left\langle Y^{(p)}\right\rangle=Y_{*}-C_{1}\langle\xi \mid \xi>0\rangle .
$$

For uniformly distributed $\xi$, the constant $C_{1}$ was evaluated as $C_{1}=2 \bar{Z}$ but its value can be different for other distributions. 
We now fix our attention to the leading point. In a competition, the leading point is always a winner but its mixing loser may overtake the leading point provided $\xi$ can be negative. The probability of overtaking is determined by $\langle H(-\xi)\rangle$, where $H(\xi)$ is the Heaviside step function: $H(\xi)=0$ for $\xi<0$ and $H(\xi)=1$ for $\xi \geqslant 0$. This results in an increase of the leading point value $Y_{*}$ by $\langle-\xi \mid \xi<0\rangle$. These considerations can be expressed by the following relation:

$$
u_{*}=\frac{C_{2}}{\Delta t}\langle-\xi H(-\xi)\rangle \text {, }
$$

where $C_{2}$ accounts for the possibility of launching particles into the region $Y>Y_{*}$ by other particles from the leading group. We derived that $C_{2}=\exp (2) / 2$ for a uniformly distributed $\xi$ (in this case $\langle-\xi H(-\xi)\rangle=m y_{*}^{2} / 2$ ).

We now consider competition with localization in physical space. In this competition, particles move in physical space according to the Ito equation

$$
\mathrm{d} x_{p}^{i}=A^{i}\left(\mathbf{x}_{p}, t\right) \mathrm{d} t+b^{i j}\left(\mathbf{x}_{p}, t\right) \mathrm{d} \omega_{p}^{j}
$$

and mixing is localized in the physical space. Here $\omega_{p}^{j}$ represents the Wiener stochastic process, while the choice of the coefficients is clarified when needed. In this case competitive mixing is allowed only between particles that are close to each other. The degree of localization can be characterized by parameter $\Lambda$ that specifies an average probability for a particle to be repeatedly mixed with the same particle. If $\Lambda$ is small $\left(\approx 1 / n_{\mathrm{p}}\right)$, then mixing is not localized. The strongest localization corresponds to $\Lambda=1$ : under these conditions particles always mix with the same partners over and over again. The limit of $\Lambda \rightarrow 1$ is quite transparent: all particles are divided into nearly permanent couples and the probability that a loser moves to a stronger position than a winner is given by $\langle H(-\xi)\rangle$. Hence, each couple would move in the direction of positive $Y$ with the speed given by (33) and $C_{2}=1$. One can see that in the case of transitive competition the effect of localization in physical space is not dramatic and results in adjustment of the constant $C_{2}=C_{2}(\Lambda)$.

\section{Intransitive competition}

Intransitive competition presumes the situation when

$$
\mathbf{y}^{(p)} \prec \mathbf{y}^{(q)} \prec \mathbf{y}^{(r)} \prec \mathbf{y}^{(p)}
$$

is possible, i.e. there is at least one set of particles $p, q$ and $r$ that satisfy (35). An example of intransitive competition is given by the well-known game of paper-scissors-rock. This game is illustrated in figure 3, where arrows point in the direction from the losers to the winners. The domain $G$ in this figure is represented by a ring with three points selected to notionally represent paper, scissors and rock. The competition in this case remains locally transitive (see the appendix) and particle systems would locally behave as discussed in the previous section. The case of strong intransitivity, when intransitive triplets can be found in any locality, is more interesting since this intransitivity can strongly affect the outcomes of the competition. The example given below demonstrates the possibility of competition with

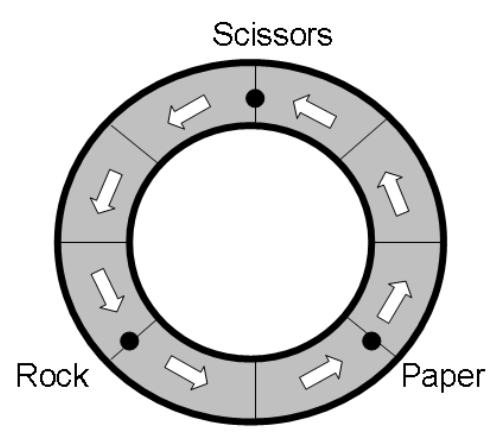

Figure 3. Example of globally intransitive and locally transitive competition. Arrows point from losers to winners and points notionally representing sissors, paper and rock are shown for illustrative purposes.

even stronger intransitive property when for any local couple $\mathbf{y}^{(p)} \prec \mathbf{y}^{(q)}$ from $G$ another particle $\mathbf{y}^{(r)}$ that satisfies (35) can be found from the same locality. Intransitivity is related to multiplicity of ranking criteria $R_{1}, \ldots, R_{k}$ with the overall winner determined by the competition index function

$$
I^{(p q)}=\sum_{i=1}^{k} w_{i} \operatorname{sign}\left(R_{i}\left(\mathbf{y}^{(p)}\right)-R_{i}\left(\mathbf{y}^{(q)}\right)\right),
$$

where $w_{i}$ are weights assigned to different criteria. The overall ranking $R_{\mu}$ can be introduced for a given reference measure $\mu$ by

$$
R_{\mu}\left(\mathbf{y}^{\circ}\right)=\mathbb{P}\left(\mathbf{y}^{(\mu)} \precsim \mathbf{y}^{\circ}\right)
$$

although this ranking does not determine the outcome of competition since $I^{(p q)}$ can be -1 even if $R_{\mu}\left(\mathbf{y}^{(p)}\right)>$ $R_{\mu}\left(\mathbf{y}^{(q)}\right)$. The overall ranking can be introduced on the basis of a fixed probability measure or with the use of a current distribution of particles as a measure. Unless specified to the contrary, we use a uniform probability measure. Practically, intransitiveness should be ubiquitously found in complex competing systems where it is difficult to establish a single ranking criterion for the winners.

An example of strongly intransitive competition (which is probably the simplest possible) is given by the following partial ranking functions: $R_{i}=y_{i}=1-Y_{i}$ where $i=1,2,3$ and $w_{i}=1$. The set of particle properties $Y_{1}, Y_{2}$ and $Y_{3}$ is assumed to satisfy the inequality $0 \leqslant Y_{i} \leqslant 1$ and the resource constraint

$$
Y_{1}+Y_{2}+Y_{3}=1
$$

The competition index function simplifies to

$$
I^{(p q)}=\sum_{i=1}^{3} \operatorname{sign}\left(Y_{i}^{(q)}-Y_{i}^{(p)}\right) .
$$

This essentially means that $\mathbf{Y}^{(p)}$ is a winner over $\mathbf{Y}^{(q)}$ when $Y_{i}^{(p)}<Y_{i}^{(q)}$ for a majority of the indices $i=1,2,3$. The competition outcomes for this case are shown in figure 4 . The domain $G$ is represented by the large triangle with parameters $Y_{1}, Y_{2}$ and $Y_{3}$ indicated for the particle $p$ whose location is shown by the black dot. The darkened areas are dominated by particle $p$ in competition, while the white areas are dominated by particle $p$. One can easily see that the particles $p, q$ and $r$ shown in the figure form an intransitive triplet (35). Properties 


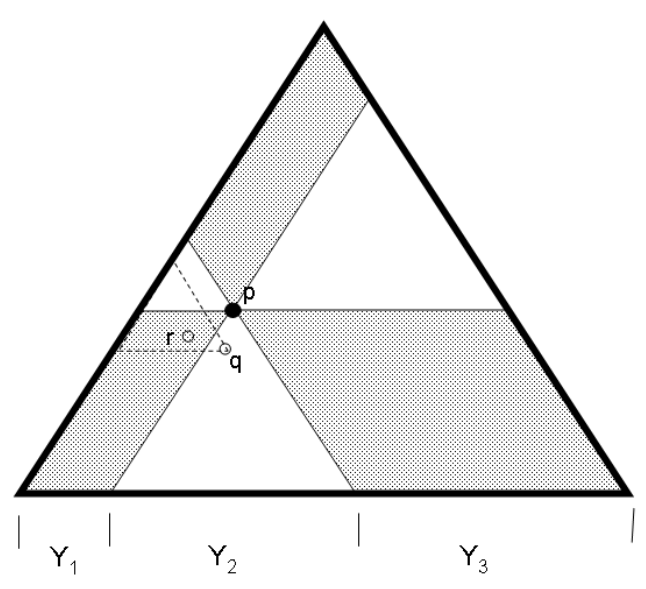

Figure 4. Intransitive competition in a two-dimensional property domain.

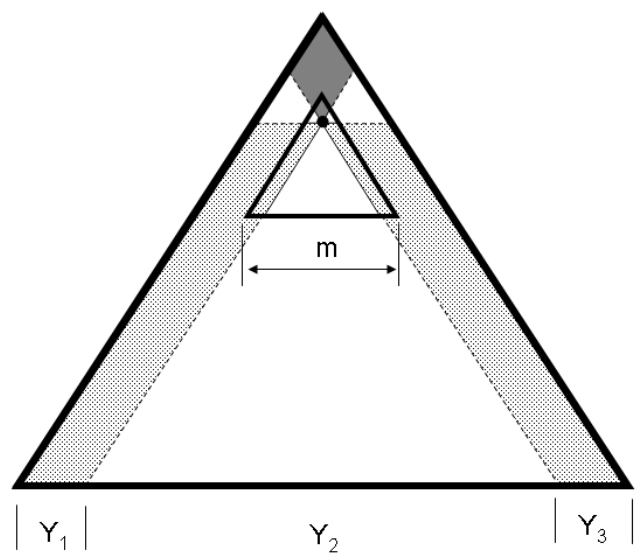

Figure 5. Mutation triangle generated from a point shown as a black dot.

of competition specified by (38) and (39) are considered in the rest of this section.

For any mixing couple, $p$ and $q$, where $q$ is a winner, the mutations for this case are given by

$$
\dot{\mathbf{Y}}^{(p)}=(1-m) \mathbf{Y}^{(q)}+m \zeta, \quad \mathbf{Y}^{(p)} \prec \mathbf{Y}^{(q)},
$$

where $\zeta$ is a random vector uniformly distributed on the triangle domain $G$. The triangle encompassing mutations from the particle near the top vertex is shown in figure 5 . For uniform distribution, the overall ranking $R$ (we omit the subscript when $\mu$ is uniform) is determined by white areas within the triangle domain $G$ in the figure and can be easily evaluated as

$$
R=Y_{1}^{2}+Y_{2}^{2}+Y_{3}^{2}
$$

The function $R$ is shown in figure 6. The strongest locations, with $R \rightarrow 1$, are at the vertices of the triangle, while the weakest point, with $R=1 / 3$ is at the center. This overall ranking influences but does not determine the outcomes of competition for the index function given by (39).

\subsection{Competitive degradation}

The effect of localization in physical space becomes crucial for intransitive competition. The example of development from the same initial conditions for different types of

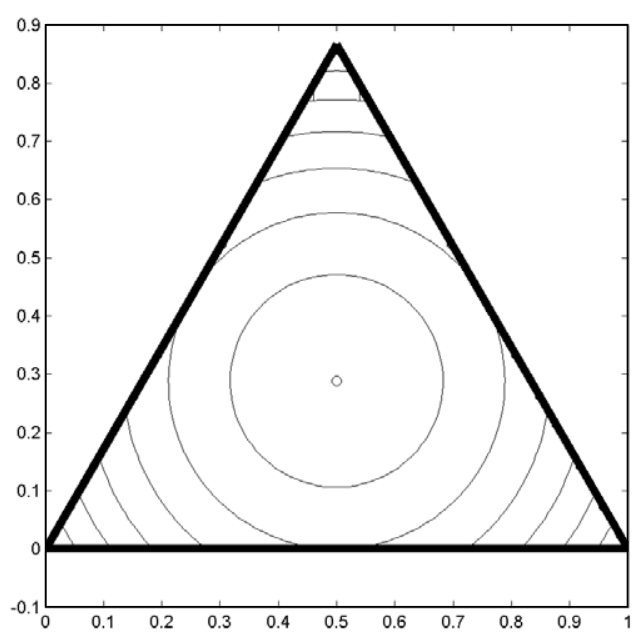

Figure 6. Overal ranking $R$ based on uniform distribution for intransitive competition.
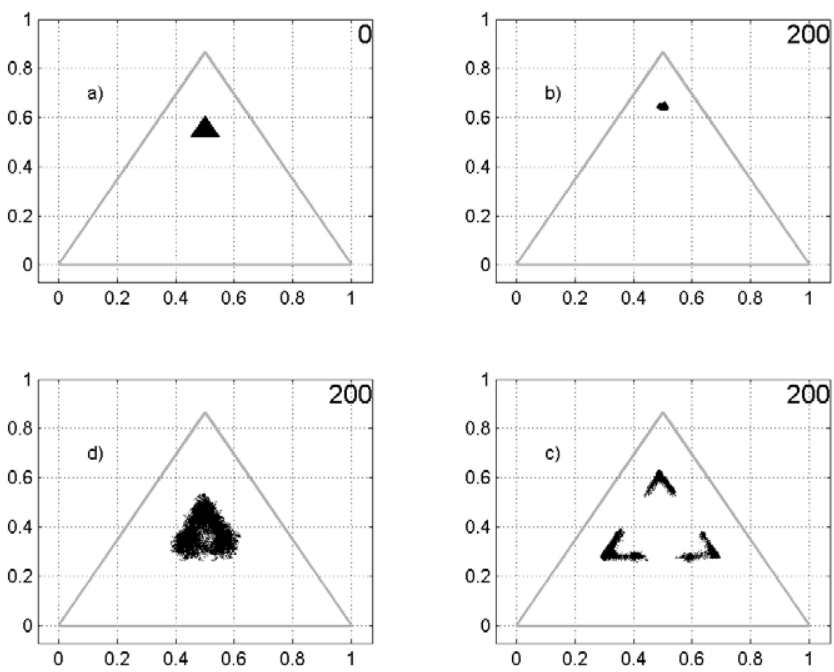

Figure 7. Simulation of 200 steps with $2^{14}$ particles for different types of competitive mixing: (a) initial conditions, (b) transitive, (c) intransitive global and (d) intransitive and localized in physical space.

competitive mixing is shown in figure 7 . In the present work, the coefficients of equation (34) are selected to represent the two-dimensional Ornstein-Uhlenbeck process. Figure 7(a) corresponds to initial conditions, while figures (b)-(d) show particle distributions after 200 steps. Calculations use $2^{14}$ particles with $m=0.015$. The competition in figure $7(\mathrm{~b})$ is transitive with $I^{(p q)}=\operatorname{sign}\left(R\left(\mathbf{Y}^{(p)}\right)-R\left(\mathbf{Y}^{(q)}\right)\right)$, where the function $R(\mathbf{Y})$ is given by (41). In this case, localized and global competitions produce very similar results and only the case with global competition $(\Lambda \approx 0)$ is shown. Note the increase in the leading point position over its initial value of 0.6. Further calculations would move particles further up towards the top strongest point at the top vertex.

Figures 7(c) and (d) show the result for intransitive competition with $I^{(p q)}$ given by (39). In figure 7(c), competition is global $(\Lambda \approx 0)$, while in figure $7(\mathrm{~d})$ competition is localized in physical space $(\Lambda \approx 0.3)$. The outcomes are very different. In the case of global competition, particles move in all directions of increasing strength towards the vertices of the triangle and continue to fiercely compete 
with each other. Localized competition results in pyramid-like structures where competition is moderated and mainly occurs between particles occupying similar ranking levels, but the strength of the structure decreases due to gradual loss of the leading positions. In localized competition the properties of particles competing with their neighbors undertake relatively small adjustments, while in global competition particles with radically different properties have to struggle for the survival of their location in the $Y$-space. Intransitive competition opens up the occasional possibility when a particle with higher overall rank $R$ loses competition to a particle with a lower overall rank $R$-this situation can be called a competitive error. Competitive degradation is an accumulation of a large number of competitive errors: each of the errors does not have any dramatic or even noticeable effect but, over a longer period of time, these errors accumulate into a decrease in competitiveness. Localized intransitive competition allows for the appearance of hierarchal structures, which, as shown in the next subsection, can exercise control over large domains with a reduced level of intra-structural competition, but have to inevitably face the effect of competitive degradation.

As in the previous section, the position of a structure is controlled by its leading group. The rank leader of the structure eventually loses to someone who has a lower rank but is still strong enough to find an occasional dominant position with respect to the leader. Competitive degradation is gradual weakening of the structures observed under localized intransitive competition. We now consider the leading particle (i.e. particle having the largest value of $R$ ) located close to one of the vertices, say vertex 2 (the top vertex of the triangle). In figure 5, this particle is shown as a black dot. The distance from the vertex is described by $y_{*}=1-Y_{2 *}$. Maximal localization $\Lambda \rightarrow 1$ is assumed. The small triangle represents the area produced by mutations originating from the dot position according to (40). The probability of competitive escalation is determined by the probability of finding a particle losing competition to the leading particle in the area represented by the small triangle above the dot. This probability can be estimated as $\sim y_{*}^{2}$, while the increase in $Y_{2}$ is proportional to $m y_{*}$. Hence, the rate of competitive escalation is determined by

$$
u_{+}=C_{3}(\Lambda) \frac{m}{\Delta t} y_{*}^{3},
$$

where $C_{3}$ represents a $\Lambda$-dependent constant. The probability of competitive degradation is determined by the probability of the losing particle landing in gray zones in the small triangle $\left(\sim y_{*}\right)$ and following mixing with this particle resulting in reduction of $Y_{2}$ by a value proportional to $m$. Hence, the rate of competitive degradation is given by

$$
u_{-}=-C_{4}(\Lambda) \frac{m}{\Delta t} y_{*}
$$

One can see that, for small $y_{*}$, the rate of degradation is dominant. If $\Lambda$ becomes small the coefficient $C_{4}$ decreases significantly to a very small value due to the low probability of repeated mixing, while $C_{3}$ somewhat increases due to the contribution to escalation from other particles in the leading group (similar to $C_{2}$ in the transitive competition case). As a result, escalation becomes dominant over degradation for global competition $\Lambda \rightarrow 0$. Note that the probability of successfully challenging the leader is very low for a randomly selected particle due to a significant difference in rank, while challenging the leader is much easier for the members of the leading group.

Competitive degradation is characterized by reduction of the average overall ranking defined by

$$
\bar{R}_{\mu}\left(\mu^{*}\right)=\mathbb{P}\left(\mathbf{y}^{(\mu)} \precsim \mathbf{y}^{\left(\mu^{*}\right)}\right),
$$

where measure $\mu^{*}$ refers to the current distribution of all particles or their leading group. The degradation affects the properties of many particles, while specific particles may increase or decrease their ranking during this process. The reference measure $\mu$ considered in this subsection is uniform within the domain and so is the mutation generator. The uniform reference measure gives realistic estimations for the overall competitive strength of particle properties. It should be mentioned, however, that definitions of competitive degradation and escalation are relativistic in intransitive systems (i.e. depend on the selected point of view represented by the reference measure). Even for obvious degradation, considered above, a 'strange' reference measure $\mu$ can be selected in a way that $\bar{R}_{\mu}$ of the leading group may increase in time. Degradation may look like escalation to some observers using 'wrong' measure $\mu$ while selecting the 'right' reference measure in complex systems may be very difficult. In complex spaces of large dimensions, escalation with respect to some parameters may be accompanied by degradation with respect to other parameters. The measure $\mu$ should be related to likely distribution of expected competitors but these expectations may change rapidly as considered in the next subsection. For example, an investment strategy that seems correct at the time of a financial boom may look quite misguided after the share market collapses. We also note that a simple measure based on the current distribution of particles $\bar{R}_{\mu^{*}}$ is not particularly useful since $\bar{R}_{\mu^{*}}\left(\mu^{*}\right)=1 / 2$ - the numbers of winners and losers are always the same in two-particle competitions.

\subsection{Leaping cycle}

In this subsection, we consider a more complicated simulation that introduces large but rare mutations, since it can be expected that from time to time a complex competing system is affected by larger than usual disturbances. Equation (40) still applies but $m$ becomes a random value, small in most cases and occasionally larger than usual (note the conceptual similarity with modification of Curl's model [16, 17]). It is important to stress that the vector $\boldsymbol{\zeta}$ is absolutely random and, as in the previous consideration, is generated with uniform distribution on the property triangle. In the presented simulations $n_{p}=$ $2^{16}$ particles were used with localization $\Lambda=0.3$. The mutation parameter was $m=0.002$ (background mutations), $m=0.8$ with probability $0.1 / n_{p}$ (reformation) and $m=0.98$ with probability $0.03 / n_{p}$ (revolution). While reformatory mutations tend to modify and sometimes strengthen existing structures, revolutionary mutations represent a wild card with unpredictable consequences.

A wide spectrum of different events can be observed in these simulations. Structures that have a pyramidal form 

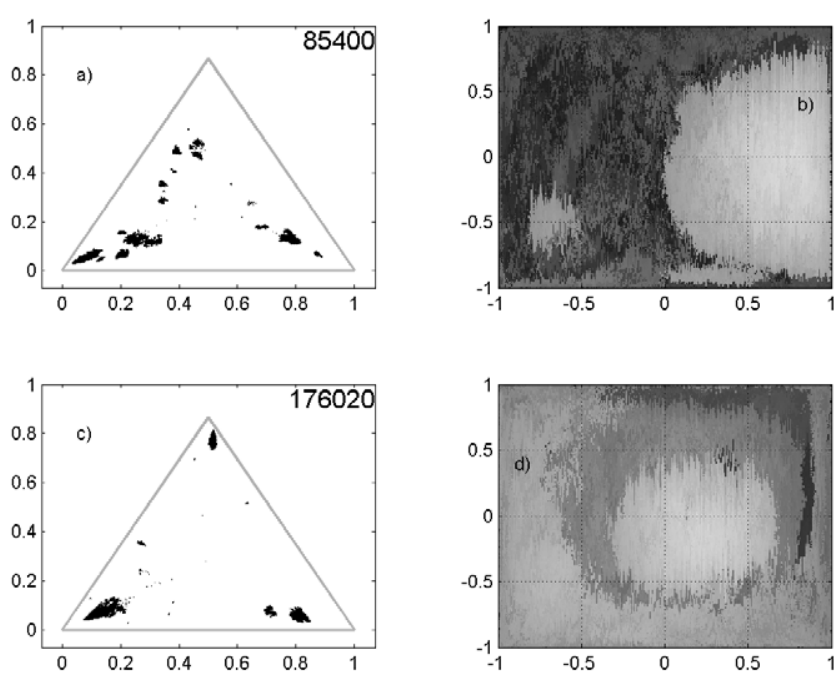

Figure 8. Selected frames of the long-time simulation for property space (left column) and physical space (right column) at 85400 steps (top row) and 176020 steps (bottom row).

in the property space and a spot-like look in the physical space appear, compete with other structures and inevitably disappear. Sometimes the whole field is dominated by a single pyramidal structure; sometimes several structures compete for the space with different levels of success and sometimes the situation becomes highly chaotic and rapidly changing. Structures can also appear under chaotic conditions but they do not achieve dominance and disintegrate quickly. The full details of distribution of particle properties in physical space can be seen only in color, but black and white figure 8 gives an illustration of observed images. The physical space, which has a Gaussian distribution of particles, is mapped into a $2 \times 2$ rectangle using the 'erf' functions. The case (a) and (b) shows two structures, large and small, taking over the rest of the area immersed in chaos. The case (c) and (d) shows a bright structure in the center taking over the area previously occupied by a darker structure. In the property space (figures 8(a) and (c)), several pyramid-like structures can be easily detected.

The hypothesis of commonality of cyclic behavior in different competitive systems was introduced in [14] citing known technological, economic, biological and historical cycles. The term 'leaping cycle' was used there to characterize generic features of this cyclic behavior [18]. If the cyclic behavior is common for competitive systems, the leaping cycle should also be observed in abstract simulations of competition. The leaping cycle is indeed clearly visible in the simulations presented here. These simulations also provide a qualification for the commonality hypothesis indicating that the cyclic behavior and increase in complexity are associated with localized intransitive competition.

The time series presented in figure 9 indicates the existence of a leaping cycle. Large structures tend to appear and quickly leap into dominance after several particularly successful mutations. The following stable period is characterized by a dominance of the successful structure, but this dominance does not last forever. The process of competitive degradation takes its toll and the dominant structure gradually weakens. Initially, this can hardly be noticed by any significant changes in the physical domain and
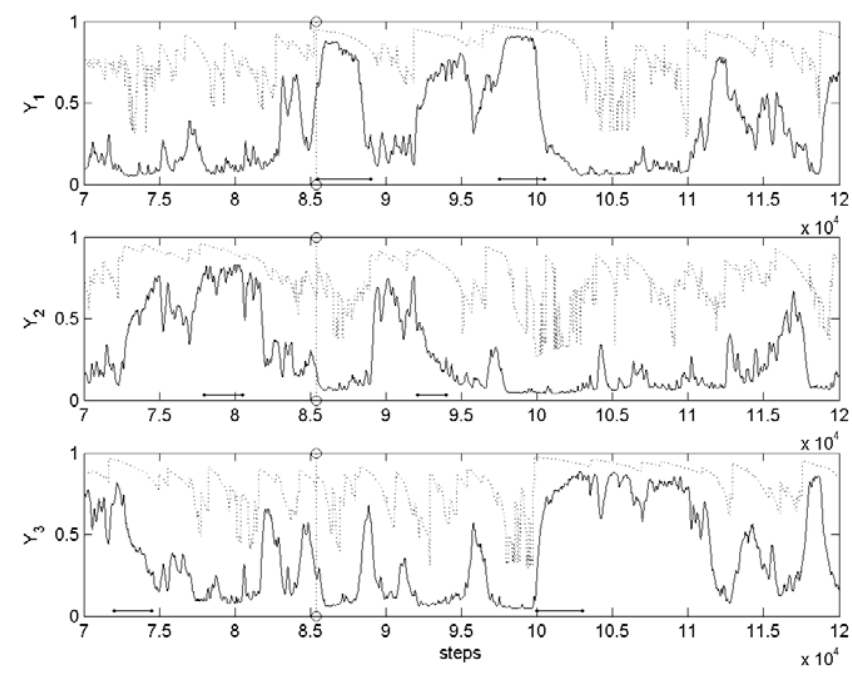

Figure 9. Property changes during the long-time simulation: $\left\langle Y_{i}\right\rangle ;---, Y_{i *}$. The vertical line shows 85400 steps. Horizontal lines show intervals used in the next figure.

the position of the dominant structure seems unshakable. In figure 9, weakening of the leading position $Y_{i *}$ is not initially accompanied by any loss of its territory and reduction of $\left\langle Y_{i}\right\rangle$. However, as the leading structure weakens further, a greater number of short-lived structures challenging the leader's domination appear but usually are successfully fended off by the leader. The process of competitive degradation can sometimes be reversed by a successful mutation reforming and invigorating the dominant structure, but competitive degradation always resumes. When the dominant structure becomes obviously weak, two outcomes are possible: (i) the domain slowly slides into chaos until/or (ii) another strong structure is born and leaps into dominance. The leaping cycle is then repeated. The characteristic duration of the leaping cycle is several thousands of steps in this example. Three extra long intervals (and many shorter ones) can be identified in figure 9. The first period is characterized by pre-eminent dominance of $Y_{2}$, the second is characterized by dominance of $Y_{1}$, and $Y_{3}$ becomes more dominant in the last period.

Equation (43) results in the following expression for the rate of competitive degradation

$$
y_{*}=\exp \left(\beta\left(S-S_{0}\right)\right)
$$

where $S$ is the number of steps, $S_{0}$ is a constant and $\beta=C_{4} m$. Equation (45) is compared in figure 10 with the simulated rate of competitive degradation from six intervals shown in figure 9 .

\section{Conclusions}

Stochastic simulations involving particles and mixing are conventionally used to model the properties of turbulent reacting flows. Conservative and non-competitive mixing is conventionally used in these simulations. This work introduces non-conservative and competitive mixing that, in principle, can be used to model a wide range of physical phenomena and represents a generic tool for the investigation of the properties of competing systems.

Analysis of competing systems can be complicated and using average quantities or conventional diffusional 


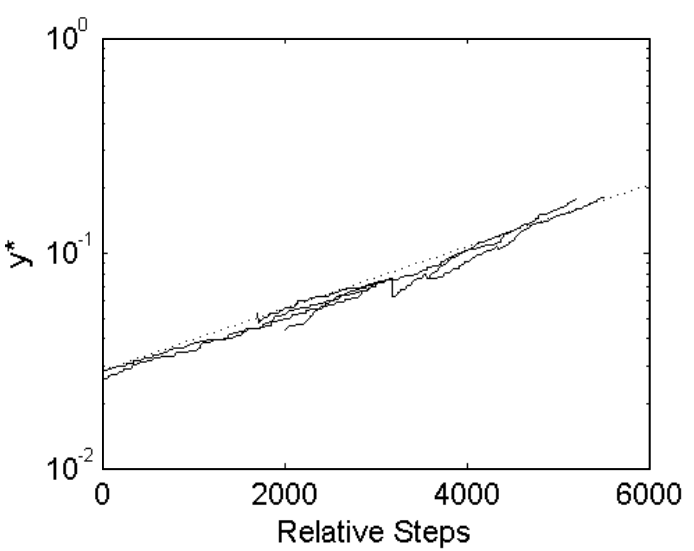

Figure 10. Rate of comperative degradation: -, results of simulation, data from six intervals shown in figure 9 ; - - equation (45) with $\beta=3.3 \times 10^{-4}$.

approximations may be inaccurate. The suggested approach is based on analysis of the vicinity of the leading point-this point is a dominant factor that strongly affects the behavior of a competing system. The rates of competitive escalation and degradation determined by this analysis are in very good agreement with the computer simulation results.

The outcomes of the competition depend upon the properties of competition-its transitiveness and localization. Transitive and global competitions tend to increase competitiveness but not complexity. Localized intransitive competition allows particles to build complicated hierarchal structures, dominate collectively and reduce the overall competition effort (this can be referred to as competitive cooperation). There is, however, a downside to this process - the strength of these structures tends to slowly decrease with time due to the process of competitive degradation. The presented simulations confirm that cyclic behavior can be expected in systems with localized intransitive competition.

\section{Acknowledgments}

D A Klimenko and K Slaughter are acknowledged for assistance with programming and simulations. The author thanks S B Pope for insightful remarks. This work was supported by the Australian Research Council.

\section{Appendix}

\section{A.1. Ranking for transitive competition}

A theorem proved more than 50 years ago by Debreu [19] states that, if subsets of $G$ specified by $\left\{\mathbf{y}^{(p)} \mid \mathbf{y}^{(p)} \precsim \mathbf{y}^{\circ}\right\}$ and

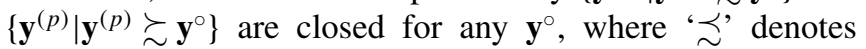
a total and transitive pre-order, then a continuous ranking function $R_{\mathrm{D}}=R_{\mathrm{D}}(\mathbf{y})$ preserving the ordering (i.e. $\mathbf{y}^{(p)} \precsim$ $\left.\mathbf{y}^{(q)} \Leftrightarrow R_{\mathrm{D}}\left(\mathbf{y}^{(p)}\right) \leqslant R_{\mathrm{D}}\left(\mathbf{y}^{(q)}\right)\right)$ can be introduced. This function is obviously not unique as, for example, $R_{\mathrm{D}}^{3}(\mathbf{y})$ introduces the same ordering. The necessity of this condition can be illustrated by the fact that $R_{\mathrm{D}}\left(\mathbf{y}^{(p)}\right) \leqslant R_{\mathrm{D}}\left(\mathbf{y}^{\circ}\right)$ and $R_{\mathrm{D}}\left(\mathbf{y}^{(p)}\right) \geqslant$ $R_{\mathrm{D}}\left(\mathbf{y}^{\circ}\right)$ define closed sets when $R_{\mathrm{D}}(\mathbf{y})$ is continuous.

Here, we use a somewhat different ranking linked to probability. Assuming that a measure $\mu$ defining probability
$\mathbb{P}$ is introduced on $G$ we specify the ranking function by $R_{\mu}\left(\mathbf{y}^{\circ}\right)=\mathbb{P}\left(\mathbf{y}^{(\mu)} \precsim \mathbf{y}^{\circ}\right)$ (where the reference measure $\mu$ is linked to the probability of particle distributions determined by mutations). It is clear that $R_{\mu}\left(\mathbf{y}^{(p)}\right)<R_{\mu}\left(\mathbf{y}^{(p)}\right) \Rightarrow \mathbf{y}^{(p)} \prec$ $\mathbf{y}^{(q)}$ and $\left.\mathbf{y}^{(p)} \simeq \mathbf{y}^{(q)} \Rightarrow R_{\mu}\left(\mathbf{y}^{(p)}\right)=R_{\mu} \mathbf{y}^{(q)}\right)$. It should be noted that this ranking can alter the ordering since it is possible that $R_{\mu}\left(\mathbf{y}^{(p)}\right)=R_{\mu}\left(\mathbf{y}^{(q)}\right)$ but $\mathbf{y}^{(p)} \prec \mathbf{y}^{(q)}$. These changes, however, are not significant and occur in areas whose measure $\mu$ is zero. The ranking function $R_{\mu}\left(\mathbf{y}^{\circ}\right)$ is, effectively, the cumulative probability $\mathbb{P}\left(\mathbf{y}^{(\mu)} \precsim \mathbf{y}^{\circ}\right)$ of the random vector $\mathbf{y}^{(\mu)}$, and $R_{\mu}\left(\mathbf{y}^{(p)}\right)=R_{\mu}\left(\mathbf{y}^{(q)}\right)$ means that the probability of particle $r$ located at $\mathbf{y}^{(p)} \prec \mathbf{y}^{(r)} \precsim \mathbf{y}^{(q)}$ is zero.

Even if the conditions of the Debreu theorem are not satisfied and the exact equivalent ranking cannot be introduced, the ranking given by $R_{\mu}\left(\mathbf{y}^{\circ}\right)=\mathbb{P}\left(\mathbf{y}^{(\mu)} \precsim \mathbf{y}^{\circ}\right)$ is practically equivalent to the original ordering from the perspective of the competition outcomes. In the opposite case when the Debreu ranking function $R_{\mathrm{D}}\left(\mathbf{y}^{\circ}\right)$ exists, the value $R_{\mathrm{D}}$ can be treated as random. The ranking $R\left(\mathbf{y}^{\circ}\right)=F_{\mathrm{D}}\left(R_{\mathrm{D}}\left(\mathbf{y}^{\circ}\right)\right)$ specified by the cumulative probability $F_{\mathrm{D}}\left(R^{\circ}\right)=\mathbb{P}\left(R_{\mathrm{D}} \leqslant\right.$ $R^{\circ}$ ) is the same as given by (11) and fully equivalent to Debreu ranking when $F_{\mathrm{D}}\left(R^{\circ}\right)$ is strictly monotonic and continuous.

\section{A.2. Types of intransitivity}

Intransitivity may appear at large scales while competition remains locally transitive. This means that there is such $\varepsilon$ that no intransitive triplets can be found in the $\varepsilon$-vicinity of every point in the domain $G$. If $G$ is compact, then a finite $\varepsilon$-grid can cover $G$ and, within each cell, competition remains transitive and can be represented by a ranking function. Although ranking functions can be different in different cells, they represent the same ranking at the cell boundaries and the ranking functions in any two neighborhood cells can be made consistent. The consistent representation of ranking functions can propagate from one cell to another and as the number of cells is finite, this procedure will eventually cover the whole domain. Whether this procedure results in an overall transitive ranking depends on the topology of the domain. For example, the competition illustrated by figure 3 is locally transitive and globally intransitive. The intransitive loop shown by the arrows can exist in this case as the loop cannot be continuously shrunk within this domain to a size smaller than $\varepsilon$. If particle competition (and distribution) is localized, then the overall behavior of the particle system would be predominantly transitive and the influence of global intransitivity would be limited.

It is also possible to have competition with locally intransitive and globally transitive properties. Indeed, let us assume that the ordering $\mathbf{y}^{(p)} \precsim \mathbf{y}^{(p)}$ is strongly intransitive (i.e. intransitive triplets (35) can be found in a small vicinity of every point). We say that particle $p$ is transitively equivalent to particle $q$ and denote this equivalence by $\mathbf{y}^{(p)} \sim_{t} \mathbf{y}^{(q)}$ when particle sets $r_{1}, \ldots, r_{n_{r}}$ and $s_{1}, \ldots, s_{n_{s}}$ exist so that

$$
\mathbf{y}^{(p)} \precsim \mathbf{y}^{\left(r_{1}\right)} \precsim \cdots \precsim \mathbf{y}^{\left(r_{n r}\right)} \precsim \mathbf{y}^{(q)}
$$

and

$$
\mathbf{y}^{(p)} \succsim \mathbf{y}^{\left(s_{1}\right)} \succsim \cdots \succsim \mathbf{y}^{\left(s_{n_{s}}\right)} \succsim \mathbf{y}^{(q)} .
$$

When $\mathbf{y}^{(p)} \sim \mathbf{y}^{(q)}$, obviously $\mathbf{y}^{(p)} \sim_{t} \mathbf{y}^{(q)}$ since equations (46) and (47) are satisfied with $n_{r}=n_{s}=0$. However, $\mathbf{y}^{(p)} \sim_{t} \mathbf{y}^{(q)}$ 
does not necessarily mean that $\mathbf{y}^{(p)} \sim \mathbf{y}^{(q)}$. It is easy to see that transitive equivalence is indeed transitive: $\mathbf{y}^{(p)} \sim_{t} \mathbf{y}^{(q)}$ and $\mathbf{y}^{(q)} \sim_{t} \mathbf{y}^{(r)}$ result in $\mathbf{y}^{(p)} \sim_{t} \mathbf{y}^{(r)}$.

We also define the binary relationship $\mathbf{y}^{(p)} \prec_{t} \mathbf{y}^{(q)}$, which means that $\mathbf{y}^{(p)} \prec \mathbf{y}^{(q)}$ and at the same time $\mathbf{y}^{(p)} \nsim_{t} \mathbf{y}^{(q)}$ It is possible to demonstrate that this relationship is transitive: $\mathbf{y}^{(p)} \prec_{t} \mathbf{y}^{(q)}$ and $\mathbf{y}^{(p)} \prec_{t} \mathbf{y}^{(r)}$ obviously result in $\mathbf{y}^{(p)} \prec_{t} \mathbf{y}^{(r)}$. Indeed, among all possibilities $\mathbf{y}^{(p)} \succ \mathbf{y}^{(r)}, \mathbf{y}^{(p)} \sim \mathbf{y}^{(r)}$ and $\mathbf{y}^{(p)} \prec \mathbf{y}^{(r)}$, only the last one is compliant with relationships $\mathbf{y}^{(p)} \prec_{t} \mathbf{y}^{(q)}$ and $\mathbf{y}^{(q)} \prec_{t} \mathbf{y}^{(r)}$, while the case of $\mathbf{y}^{(p)} \prec \mathbf{y}^{(r)}$ and $\mathbf{y}^{(p)} \sim_{t} \mathbf{y}^{(r)}$ should be eliminated for the same reason. The only remaining possibility is $\mathbf{y}^{(p)} \prec_{t} \mathbf{y}^{(r)}$.

The new binary relation splits all property states into classes of equivalence with transitive ordering between these classes, which is consistent with the original definition of the winners and losers (i.e. $\mathbf{y}^{(p)} \prec_{t} \mathbf{y}^{(r)}$ requires that $\mathbf{y}^{(p)} \prec$ $\left.\mathbf{y}^{(r)}\right)$. Thus, competition within each class can be omitted from consideration while each class can receive its equivalent rank determining the outcomes of the global competition between the classes. Once the winning class is determined, the competition will continue locally within this class according to intransitive rules.

The examples of this section illustrate that in complex competition, transitive and intransitive competition rules can be combined hierarchically (like Russian dolls).

\section{References}

[1] Pope S B 1985 Pdf methods for turbulent reactive flows Prog. Energy Combust. Sci. 11 119-92

[2] Kuznetsov V R and Sabelnikov V A 1990 Turbulence and Combustion (New York: Hemisphere)

[3] Dopazo C 1994 Recent developments in Pdf methods Turbulent Reacting Flows ed P A Libby and F A Williams (London: Academic) chapter 7, pp 375-474
[4] Pope S B 2000 Turbulent Flows (Cambridge: Cambridge University Press)

[5] Fox R 2003 Computational Models for Turbulent Reacting Flows (Cambridge: Cambridge University Press)

[6] Heinz S 2003 Statistical Mechanics of Turbulent Flows (Berlin: Springer)

[7] Pitsch H 2006 Large-eddy simulations of turbulent combustion Annu. Rev. Fluid Mech. 38 453-82

[8] Haworth D 2010 Progress in probability density function methods for turbulent reacting flows Prog. Energy Combust. Sci. 36 168-259

[9] Dreeben T D and Pope S B 1997 Probability density function and Reynolds-stress modeling of near-wall turbulent flows Phys. Fluids 9 154-63

[10] Klimenko A Y 2007 On simulating scalar transport by mixing between Lagrangian particles Phys. Fluids 19031702

[11] Cleary M J and Klimenko A Y 2009 A generalised multiple mapping conditioning approach for turbulent combustion Flow, Turbul. Combust. 82 477-91

[12] Klimenko A Y and Cleary M J 2010 Convergence to a model in sparse-Lagrangian FDF simulations Flow, Turbul. Combust. (Special issue dedicated to 60th birthday of S B Pope) submitted

[13] Klimenko A Y 2009 Lagrangian particles with mixing. I. simulating scalar transport Phys. Fluids 21065101

[14] Klimenko A Y 2008 Technological cycles and their impact on science, engineering and engineering education Int. J. Technol. Knowl. Soc. 4 11-8

[15] Curl R L 1963 Dispersed phase mixing. I. AIChE J. 9175

[16] Janicka J, Kolbe W and Kollmann W 1979 Closure of the transport equation for the probability density function of scalar fields J. Non-Equilib. Thermodyn. 4 47-66

[17] Lindstedt R P, Louloudi S A, Driscoll J J and Sick V 2004 Finite rate chemistry effects in turbulent reacting flows Flow, Turbul. Combust. 72 407-26

[18] Klimenko A Y 2010 Computer simulations of abstract competition Proc. Int. Conf. on Complexity, Informatics and Cybernetics (IMCIC) vol 1, pp 97-102

[19] Debreu G 1954 Presentation of a preference ordering by a numerical function Decision Process ed R M Thrall, $\mathrm{C} \mathrm{H}$ Coombs and R L Davis (New York: Wiley) pp 159-165 